NASA/CR-1998-208521

$1 N-24$

Micromechanical Prediction of the Effective Coefficients of Thermo-Piezoelectric Multiphase Composites

Jacob Aboudi

Tel-Aviv University, Ramat-Aviv, Israel 
The NASA STI Program Office ... in Profile

Since its founding, NASA has been dedicated to the advancement of aeronautics and space science. The NASA Scientific and Technical Information (STI) Program Office plays a key part in helping NASA maintain this important role.

The NASA STI Program Office is operated by Langley Research Center, the Lead Center for NASA's scientific and technical information. The NASA STI Program Office provides access to the NASA STI Database, the largest collection of aeronautical and space science STI in the world. The Program Office is also NASA's institutional mechanism for disseminating the results of its research and development activities. These results are published by NASA in the NASA STI Report Series, which includes the following report types:

- TECHNICAL PUBLICATION. Reports of completed research or a major significant phase of research that present the results of NASA programs and include extensive data or theoretical analysis. Includes compilations of significant scientific and technical data and information deemed to be of continuing reference value. NASA's counterpart of peerreviewed formal professional papers but has less stringent limitations on manuscript length and extent of graphic presentations.

- TECHNICAL MEMORANDUM. Scientific and technical findings that are preliminary or of specialized interest, e.g., quick release reports, working papers, and bibliographies that contain minimal annotation. Does not contain extensive analysis.

- CONTRACTOR REPORT. Scientific and technical findings by NASA-sponsored contractors and grantees.
- CONFERENCE PUBLICATION. Collected papers from scientific and technical conferences, symposia, seminars, or other meetings sponsored or cosponsored by NASA.

- SPECIAL PUBLICATION. Scientific, technical, or historical information from NASA programs, projects, and missions, often concerned with subjects having substantial public interest.

- TECHNICAL TRANSLATION. Englishlanguage translations of foreign scientific and technical material pertinent to NASA's mission.

Specialized services that complement the STI Program Office's diverse offerings include creating custom thesauri, building customized data bases, organizing and publishing research results ... even providing videos.

For more information about the NASA STI Program Office, see the following:

- Access the NASA STI Program Home Page at http://www.sti.nasa.gov

- E-mail your question via the Internet to help@sti.nasa.gov

- Fax your question to the NASA Access Help Desk at (301) 621-0134

- Telephone the NASA Access Help Desk at (301) 621-0390

- Write to: NASA Access Help Desk NASA Center for AeroSpace Information 7121 Standard Drive Hanover, MD 21076 
NASA/CR-1998-208521

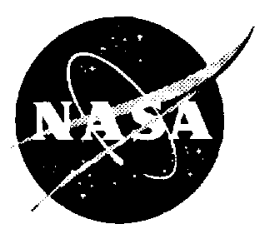

\section{Micromechanical Prediction of the Effective Coefficients of Thermo-Piezoelectric Multiphase Composites}

Jacob Aboudi

Tel-Aviv University, Ramat-Aviv, Israel

Prepared under Contract NAS3-97190

National Aeronautics and

Space Administration

Lewis Research Center 
Available from

NASA Center for Aerospace Information 7121 Standard Drive Hanover, MD 21076

Price Code: A03
National Technical Information Service 5285 Port Royal Road Springfield, VA 22100 Price Code: $\mathrm{A} 03$ 


\title{
Micromechanical Prediction of The Effective Coefficients of Thermo-Piezoelectric Multiphase Composites
}

\author{
Jacob Aboudi \\ Tel-Aviv University, Israel
}

\begin{abstract}
The micromechanical generalized method of cells model is employed for the prediction of the effective elastic, piezoelectric, dielectric, pyroelectric and thermal-expansion constants of multiphase composites with embedded piezoelectric materials. The predicted effective constants are compared with other micromechanical methods available in the literature and good agreements are obtained.
\end{abstract}

\section{Introduction}

Piezoelectric materials are dielectrics which exhibit significant material deformations in response to an applied electric field, and produce electric polarization in response to applied mechanical loadings. Piezoelectric materials are used as sensors and actuators, thus forming important ingredients in smart material systems. Detailed discussion and numerous references for piezoelectric sensors and actuators can be found in the book by Gandhi and Thompson (1992). A concentric cylinder model that couples elastic, electric, magnetic and thermal fields has been presented by Carman et al(1995).

It is possible to combine piezoelectric materials with passive materials to form various types of piezoelectric composite systems. For example one can disperse piezoelectric particles into epoxy resin, or embed piezoelectric wires into epoxy resin. Other composite systems 
are polymer filled holes in solid piezoelectric ceramic matrices, and porous piezoelectric ceramics. Alternatively, one can generate smart materials that consist of fibrous composites with embedded piezoelectric actuators and sensors. Discussions of various applications of piezoceramic-polymer composites can be found in the book by Moulson and Herbert (1990). In all these cases the overall behavior of these composites can be determined by a suitable micromechanical model, see Dunn and Taya (1993) and Dunn (1993) for example. Jain and Sirkis (1994) considered the issue of damage caused by the growth of voids in piezoelectric ceramics.

The method of cells and its generalization, referred to as the generalized method of cells (GMC), is an approximate analytical micromechanical model which is capable of predicting the overall behavior of long-fiber, short-fiber and particulate multiphase composites from the knowledge of the properties of the individual phases. A review of the method of cells followed by a monograph were given by Aboudi $(1989,1991)$. This review has been recently updated by Aboudi (1996), where critical assessments of the method and its application by various researchers were outlined. As documented, many types of composites (e.g. thermoelastic, viscoelastic, nonlinear elastic and viscoplastic) were analyzed by the method and the reliability of the predictions were demonstrated under many circumstances.

In the present paper, GMC is employed for the prediction of the overall behavior of multiphase composites that include one or several types of piezoelectric materials. By imposing mechanical equilibrium and Maxwell's charge equation in the constituent regions it is possible, in conjunction with the continuity of mechanical displacements, tractions, electric potential and electric displacements, to establish the electro-mechanical concentration tensor that relates the local field in terms of the global one. This readily enables the derivation of all effective constants of the multiphase piezoelectric material. The reliability of the predicted effective constants is examined by comparisons with other results available in the literature.

As in previous investigations where GMC was used to develop the macroscale constitutive equation and supplied as input to analyze composite structures, the derived constitutive relations given in this paper can be used to investigate the behavior of composite structures with piezoelectric components. Piezoelectric composite plates were recently considered by Tauchert(1992) and Jonnalagadda et al(1994). Thus the proposed methodology can be 
employed to perform thermo-electro-mechanical micro-to-macro analysis of composite structures based solely on the properties of the individual phases.

\section{Micromechanical Analysis}

\subsection{Model description}

Consider a multiphase composite material in which some or all phases are modeled as thermopiezoelectric materials. It is assumed that the composite possesses a periodic structure such that a repeating cell can be defined. In Fig. 1, such a repeating cell is shown which consists of $N_{\alpha} N_{\beta} N_{\gamma}$ rectangular parallelepiped subcells. The volume of each subcell is $d_{\alpha} h_{\beta} l_{\gamma}$, where $\alpha, \beta, \gamma$ are running indices $\alpha=1, \cdots, N_{\alpha} ; \beta=1, \cdots, N_{\beta} ; \gamma=1, \cdots, N_{\gamma}$ in the three orthogonal directions, respectively. The volume of the repeating cell is $d h l$ where

$$
d=\sum_{\alpha=1}^{N_{\alpha}} d_{\alpha}, \quad h=\sum_{\beta=1}^{N_{\beta}} h_{\beta}, l=\sum_{\gamma=1}^{N_{\gamma}} l_{\gamma}
$$

Any subcell can be filled in general by thermo-piezoelectric materials. Piezoelectric unidirectional long-fiber composites, short-fiber composites, porous materials, and laminated materials are obtained by a proper selection of the geometrical dimensions of the subcells and with an appropriate material fillings.

The micromechanical model employs a first order expansion of the displacement in the subcell $(\alpha \beta \gamma)$ in terms of the local coordinates $\left(\bar{x}_{1}^{(\alpha)}, \bar{x}_{2}^{(\beta)}, \bar{x}_{3}^{(\gamma)}\right)$ located at the center of the subcell.

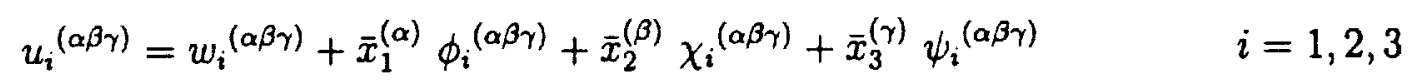

where $w_{i}{ }^{(\alpha \beta \gamma)}$ are the displacement components at the center of the subcell, and $\phi_{i}{ }^{(\alpha \beta \gamma)}$, $\chi_{i}{ }^{(\alpha \beta \gamma)}$ and $\psi_{i}{ }^{(\alpha \beta \gamma)}$ are the microvariables that characterize the linear dependence of the displacement $u_{i}^{(\alpha \beta \gamma)}$ on the local coordinates $\bar{x}_{1}^{(\alpha)}, \bar{x}_{2}^{(\beta)}, \bar{x}_{3}^{(\gamma)}$. In eqn.(2) and the sequel, repeated Greek letters do not imply summation.

Similarly the electric potential $\xi^{(\alpha \beta \gamma)}$ (voltage) is expanded linearly in terms of the local coordinates of the subcell:

$$
\xi^{(\alpha \beta \gamma)}=\xi_{0}^{(\alpha \beta \gamma)}+\bar{x}_{1}^{(\alpha)} \xi_{1}^{(\alpha \beta \gamma)}+\bar{x}_{2}^{(\beta)} \xi_{2}^{(\alpha \beta \gamma)}+\bar{x}_{3}^{(\gamma)} \xi_{3}^{(\alpha \beta \gamma)}
$$


The components of the small strain tensor $\epsilon_{i j}^{(\alpha \beta \gamma)}$ are given by

$$
\epsilon_{i j}^{(\alpha \beta \gamma)}=\frac{1}{2}\left(\partial_{i} u_{j}^{(\alpha \beta \gamma)}+\partial_{j} u_{i}^{(\alpha \beta \gamma)}\right) \quad i, j=1,2,3
$$

where $\partial_{1}=\partial / \partial \bar{x}_{1}^{(\alpha)}, \partial_{2}=\partial / \partial \bar{x}_{2}^{(\beta)}$ and $\partial_{3}=\partial / \partial \bar{x}_{3}^{(\gamma)}$.

The components of the electrical field $E_{i}^{(\alpha \beta \gamma)}$ in the subcell are obtained from the potential $\xi^{(\alpha \beta \gamma)}$ via

$$
E_{i}^{(\alpha \beta \gamma)}=-\partial_{i} \xi^{(\alpha \beta \gamma)}
$$

The constitutive equations that govern the interaction of elastic, electric and thermal fields in a piezoelectric medium (that fills the subcell $(\alpha \beta \gamma)$ ) are given by (see Parton and Kudryavtesev (1988) for example):

$$
\sigma_{i j}^{(\alpha \beta \gamma)}=C_{i j k l}^{(\alpha \beta \gamma)} \epsilon_{k l}^{(\alpha \beta \gamma)}-e_{k i j}^{(\alpha \beta \gamma)} E_{k}^{(\alpha \beta \gamma)}-\Lambda_{i j}^{(\alpha \beta \gamma)} \Delta T
$$

where $\sigma_{i j}^{(\alpha \beta \gamma)}$ are the stress components induced in the material in the subcell, and $C_{i j k l}^{(\alpha \beta \gamma)}$, $e_{k i j}^{(\alpha \beta \gamma)}$, and $\Lambda_{i j}^{(\alpha \beta \gamma)}$ denote the fourth order elastic stiffness tensor, the third order piezoelectric tensor and the second order thermal stress tensor of the material in the subcell, respectively. The temperature change from a reference temperature is denoted by $\Delta T$. In addition the electric displacement vector is given by the relation

$$
D_{i}^{(\alpha \beta \gamma)}=e_{i k l}^{(\alpha \beta \gamma)} \epsilon_{k l}^{(\alpha \beta \gamma)}+\lambda_{i k}^{(\alpha \beta \gamma)} E_{k}^{(\alpha \beta \gamma)}+p_{i}^{(\alpha \beta \gamma)} \Delta T
$$

where $\lambda_{i k}^{(\alpha \beta \gamma)}$ and $p_{i}^{(\alpha \beta \gamma)}$ are the second order dielectric tensor and the pyroelectric vector, respectively.

With the linear expansions of the displacements and the potential given by eqns.(2) and (3) the static equilibrium of the material within the subcell $(\alpha \beta \gamma)$ is satisfied, namely

$$
\sigma_{i j, j}^{(\alpha \beta \gamma)}=0
$$

and

$$
D_{i, i}^{(\alpha \beta \gamma)}=0
$$

Let the vectors $\mathrm{X}^{(\boldsymbol{\alpha} \beta \gamma)}$ and $\mathrm{Y}^{(\boldsymbol{\alpha} \beta \gamma)}$ be defined as follows:

$$
\mathbf{X}^{(\alpha \beta \gamma)}=\left[\epsilon_{11}, \epsilon_{22}, \epsilon_{33}, 2 \epsilon_{23}, 2 \epsilon_{13}, 2 \epsilon_{12},-E_{1},-E_{2},-E_{3}\right]^{(\alpha \beta \gamma)}
$$




$$
\mathbf{Y}^{(\alpha \beta \gamma)}=\left[\sigma_{11}, \sigma_{22}, \sigma_{33}, \sigma_{23}, \sigma_{13}, \sigma_{12}, D_{1}, D_{2}, D_{3}\right]^{(\alpha \beta \gamma)}
$$

By excluding the temperature effects in the constitutive equations (6)-(7), one can rewrite these equations in the compact matrix form:

$$
\mathbf{Y}^{(\alpha \beta \gamma)}=\mathbf{Z}^{(\alpha \beta \gamma)} \mathbf{X}^{(\alpha \beta \gamma)}
$$

where the 9 th order coefficient matrix $\mathbf{Z}^{(\alpha \beta \gamma)}$ has the following form

$$
Z^{(\alpha \beta \gamma)}=\left[\begin{array}{ll}
C^{(\alpha \beta \gamma)} & e^{t(\alpha \beta \gamma)} \\
e^{(\alpha \beta \gamma)} & -\lambda^{(\alpha \beta \gamma)}
\end{array}\right]
$$

In this equation the square matrix $C^{(\alpha \beta \gamma)}$ of the 6 th-order represents the 4 th order stiffness tensor, $\mathrm{e}^{\mathrm{t}(\alpha \beta \gamma)}$ denotes the transpose of the rectangular 3 by 6 matrix $\mathrm{e}^{(\alpha \beta \gamma)}$ that represents the corresponding third order piezoelectric tensor, and $\lambda^{(\alpha \beta \gamma)}$ is a square matrix of order 3 that corresponds to the dielectric tensor.

The volume average of the stresses $\bar{\sigma}_{i j}$ and electric displacements $\bar{D}_{i}$ in the entire repeating cell (namely in the composite) is given by

$$
\overline{\mathbf{Y}}=\frac{1}{d h l} \sum_{\alpha=1}^{N_{\alpha}} \sum_{\beta=1}^{N_{\beta}} \sum_{\gamma=1}^{N_{\gamma}} d_{\alpha} h_{\beta} l_{\gamma} \mathbf{Y}^{(\alpha \beta \gamma)}
$$

and similarly, that of the strains $\bar{\epsilon}_{i j}$ and electric field components $\bar{E}_{i}$ are

$$
\overline{\mathbf{X}}=\frac{1}{d h l} \sum_{\alpha=1}^{N_{\alpha}} \sum_{\beta=1}^{N_{\beta}} \sum_{\gamma=1}^{N_{\gamma}} d_{\alpha} h_{\beta} l_{\gamma} \mathbf{X}^{(\alpha \beta \gamma)}
$$

\subsection{Interfacial continuity of displacements}

Imposition of displacement continuity conditions at the various subcell interfaces within the repeating cell, and at the interfaces between neighboring repeating cells provide the following $N_{\alpha} N_{\beta}+N_{\alpha} N_{\gamma}+N_{\beta} N_{\gamma}+N_{\alpha}+N_{\beta}+N_{\gamma}$ relations between the volume-averaged subcell strains and composite strains (Aboudi(1995)):

$$
\sum_{\alpha=1}^{N_{\alpha}} d_{\alpha} \epsilon_{11}^{(\alpha \beta \gamma)}=d \bar{\epsilon}_{11}, \quad \beta=1, \cdots, N_{\beta} \quad ; \quad \gamma=1, \cdots, N_{\gamma}
$$




$$
\begin{array}{cc}
\sum_{\beta=1}^{N_{\beta}} h_{\beta} \epsilon_{22}^{(\alpha \beta \gamma)}=h \bar{\epsilon}_{22}, & \alpha=1, \cdots, N_{\alpha} ; \gamma=1, \cdots, N_{\gamma} \\
\sum_{\gamma=1}^{N_{\gamma}} l_{\gamma} \epsilon_{33}^{(\alpha \beta \gamma)}=l \bar{\epsilon}_{33}, \quad \alpha=1, \cdots, N_{\alpha} ; \beta=1, \cdots, N_{\beta} \\
\sum_{\beta=1}^{N_{\beta}} \sum_{\gamma=1}^{N_{\gamma}} h_{\beta} l_{\gamma} \epsilon_{23}^{(\alpha \beta \gamma)}=h l \bar{\epsilon}_{23}, & \alpha=1, \cdots, N_{\alpha} \\
\sum_{\alpha=1}^{N_{\alpha}} \sum_{\gamma=1}^{N_{\gamma}} d_{\alpha} l_{\gamma} \epsilon_{13}^{(\alpha \beta \gamma)}=d l \bar{\epsilon}_{13}, & \beta=1, \cdots, N_{\beta} \\
\sum_{\alpha=1}^{N_{\alpha}} \sum_{\beta=1}^{N_{\beta}} d_{\alpha} h_{\beta} \epsilon_{12}^{(\alpha \beta \gamma)}=d h \bar{\epsilon}_{12}, & \gamma=1, \cdots, N_{\gamma}
\end{array}
$$

\subsection{Interfacial continuity of tractions}

The continuity of tractions at the interfaces (both between subcells and repeating cells) provide the following $6 N_{\alpha} N_{\beta} N_{\gamma}-\left(N_{\alpha} N_{\beta}+N_{\alpha} N_{\gamma}+N_{\beta} N_{\gamma}\right)-\left(N_{\alpha}+N_{\beta}+N_{\gamma}\right)$ conditions.

$$
\begin{gathered}
\sigma_{11}^{(\alpha \beta \gamma)}=\sigma_{11}^{(\hat{\alpha} \beta \gamma)} \quad \alpha=1, \cdots, N_{\alpha}-1 ; \beta=1, \cdots, N_{\beta} ; \gamma=1, \cdots, N_{\gamma} \\
\sigma_{22}^{(\alpha \beta \gamma)}=\sigma_{22}^{(\alpha \hat{\beta} \gamma)} \quad \alpha=1, \cdots, N_{\alpha} ; \beta=1, \cdots, N_{\beta}-1 ; \gamma=1, \cdots, N_{\gamma} \\
\sigma_{33}^{(\alpha \beta \gamma)}=\sigma_{33}^{(\alpha \beta \hat{\gamma})} \quad \alpha=1, \cdots, N_{\alpha} ; \beta=1, \cdots, N_{\beta} ; \gamma=1, \cdots, N_{\gamma}-1 \\
\sigma_{23}^{(\alpha \beta \gamma)}=\sigma_{23}^{(\alpha \hat{\beta} \gamma)} \quad \alpha=1, \cdots, N_{\alpha} ; \beta=1, \cdots, N_{\beta}-1 ; \gamma=1, \cdots, N_{\gamma} \\
\sigma_{32}^{(\alpha \beta \gamma)}=\sigma_{32}^{(\alpha \beta \hat{\gamma})} \quad \alpha=1, \cdots, N_{\alpha} ; \beta=N_{\beta} ; \gamma=1, \cdots, N_{\gamma}-1 \\
\sigma_{13}^{(\alpha \beta \gamma)}=\sigma_{13}^{(\hat{\alpha} \beta \gamma)} \quad \alpha=1, \cdots, N_{\alpha}-1 ; \beta=1, \cdots, N_{\beta} ; \gamma=1, \cdots, N_{\gamma}
\end{gathered}
$$




$$
\begin{gathered}
\sigma_{31}^{(\alpha \beta \gamma)}=\sigma_{31}^{(\alpha \beta \hat{\gamma})} \quad \alpha=N_{\alpha} ; \beta=1, \cdots, N_{\beta} ; \gamma=1, \cdots, N_{\gamma}-1 \\
\sigma_{12}^{(\alpha \beta \gamma)}=\sigma_{12}^{(\alpha \beta \gamma)} \quad \alpha=1, \cdots, N_{\alpha}-1 ; \beta=1, \cdots, N_{\beta} ; \gamma=1, \cdots, N_{\gamma} \\
\sigma_{21}^{(\alpha \beta \gamma)}=\sigma_{21}^{(\alpha \hat{\beta} \gamma)} \quad \alpha=N_{\alpha} ; \beta=1, \cdots, N_{\beta}-1 ; \gamma=1, \cdots, N_{\gamma}
\end{gathered}
$$

where $\hat{\alpha}, \hat{\beta}$ and $\hat{\gamma}$ are defined in the following manner,

$$
\begin{gathered}
\hat{\alpha}= \begin{cases}\alpha+1 & \alpha<N_{\alpha} \\
1 & \alpha=N_{\alpha}\end{cases} \\
\hat{\beta}= \begin{cases}\beta+1 & \beta<N_{\beta} \\
1 & \beta=N_{\beta}\end{cases} \\
\hat{\gamma}= \begin{cases}\gamma+1 & \gamma<N_{\gamma} \\
1 & \gamma=N_{\gamma}\end{cases}
\end{gathered}
$$

Note the definition for $\hat{\alpha}$, for example, ensures that for $\alpha<N_{\alpha}$ the neighboring subcell in the $x_{1}$ - direction is the one labelled by $(\alpha+1, \beta, \gamma)$ within the repeating volume element, whereas for $\alpha=N_{\alpha}$ the neighboring subcell is within the next repeating volume element whose first subcell is $(1 \beta \gamma)$. A similar interpretation for $\hat{\beta}$ and $\hat{\gamma}$ can be made.

\subsection{Interfacial continuity of electric potential}

The continuity of the electric potential at the interfaces within the repeating cell, and between neighboring cells provides $N_{\alpha} N_{\beta}+N_{\alpha} N_{\gamma}+N_{\beta} N_{\gamma}$ relations as follows:

$$
\begin{array}{ll}
\sum_{\alpha=1}^{N_{\alpha}} d_{\alpha} E_{1}^{(\alpha \beta \gamma)}=d \bar{E}_{1}, & \beta=1, \cdots, N_{\beta} ; \gamma=1, \cdots, N_{\gamma} \\
\sum_{\beta=1}^{N_{\beta}} h_{\beta} E_{2}^{(\alpha \beta \gamma)}=h \bar{E}_{2}, & \alpha=1, \cdots, N_{\alpha} ; \gamma=1, \cdots, N_{\gamma}
\end{array}
$$




$$
\sum_{\gamma=1}^{N_{\gamma}} l_{\gamma} E_{3}^{(\alpha \beta \gamma)}=l \bar{E}_{3}, \quad \alpha=1, \cdots, N_{\alpha} ; \beta=1, \cdots, N_{\beta}
$$

where eqn. (5) and the fact that the average potential gradients are given by

$$
\frac{\partial \xi_{0}{ }^{(\alpha \beta \gamma)}}{\partial x_{i}}=\bar{\xi}_{0, i}=-\bar{E}_{i} \quad i=1,2,3
$$

for all $\alpha, \beta$ and $\gamma$ have been employed in eqns. (31)-(33).

\subsection{Interfacial continuity of the electric displacements}

Finally, the additional $3 N_{\alpha} N_{\beta} N_{\gamma}-\left(N_{\alpha} N_{\beta}+N_{\alpha} N_{\gamma}+N_{\beta} N_{\gamma}\right)$ required relations are obtained by imposing continuity conditions on the electric displacements $D_{i}$ (which ensures that they are continuous at the interfaces in the ith directions), that is,

$$
\begin{aligned}
& D_{1}^{(\alpha \beta \gamma)}=D_{1}^{(\hat{\alpha} \beta \gamma)} \quad \alpha=1, \cdots, N_{\alpha}-1 ; \beta=1, \cdots, N_{\beta} ; \gamma=1, \cdots, N_{\gamma} \\
& D_{2}^{(\alpha \hat{\beta} \gamma)}=D_{2}^{(\alpha \hat{\beta} \gamma)} \quad \alpha=1, \cdots, N_{\alpha} ; \beta=1, \cdots, N_{\beta}-1 ; \gamma=1, \cdots, N_{\gamma} \\
& D_{3}^{(\alpha \beta \gamma)}=D_{3}^{(\alpha \beta \hat{\gamma})} \quad \alpha=1, \cdots, N_{\alpha} ; \beta=1, \cdots, N_{\beta} ; \gamma=1, \cdots, N_{\gamma}-1
\end{aligned}
$$

where $\hat{\alpha}, \hat{\beta}$ and $\hat{\gamma}$ are defined as before.

\subsection{Overall constitutive equations}

The combination of the aforementioned continuity relations (16)-(37) forms a system of $9 N_{\alpha} N_{\beta} N_{\gamma}$ equations in the $9 N_{\alpha} N_{\beta} N_{\gamma}$ subcell unknowns. These unknowns can be combined and represented by a single vector as follows.

$$
\mathrm{X}_{s}=\left(\mathrm{X}^{(111)}, \mathrm{X}^{(112)}, \ldots, \mathrm{X}^{\left(N_{\alpha} N_{\beta} N_{\gamma}\right)}\right)
$$

This system of equations can be represented in the form

$$
\overline{\mathrm{A}} \mathrm{X}_{\mathbf{s}}=\mathbf{K} \overline{\mathrm{X}}
$$


where $\tilde{\mathrm{A}}$ is the square matrix of coefficients of order $9 N_{\alpha} N_{\beta} N_{\gamma}$,

$$
\overline{\mathbf{X}}=\left(\bar{\epsilon}_{11}, \bar{\epsilon}_{22}, \bar{\epsilon}_{33}, 2 \bar{\epsilon}_{23}, 2 \bar{\epsilon}_{13}, 2 \bar{\epsilon}_{12},-\bar{E}_{1},-\bar{E}_{2},-\bar{E}_{3}\right)
$$

being the average strain and electric field vector, and $\mathrm{K}$ is a rectangular matrix of the order of $9 N_{\alpha} N_{\beta} N_{\gamma}$ by 9 , that involves the geometrical properties of the subcells. Note $\tilde{\mathrm{A}}$ and $\mathrm{K}$ consist of the following submatrices:

$$
\tilde{\mathbf{A}}=\left[\begin{array}{c}
\mathbf{A}_{M} \\
\mathbf{A}_{G} \\
\mathbf{A}_{E}
\end{array}\right], \quad \mathbf{K}=\left[\begin{array}{l}
0 \\
\mathbf{J} \\
0
\end{array}\right]
$$

where $A_{M}$, involves the elastic mechanical properties of the material, $A_{G}$ the geometrical dimensions of the subcells, $A_{E}$ the electrical properties, and $J$ the dimensions of the repeating cell.

The solution of eqn.(39) yields the strain and electric field components in all subcells in the form

$$
\mathbf{X}_{\mathbf{s}}=\mathbf{A} \overline{\mathbf{X}}
$$

where

$$
\mathbf{A}=\overline{\mathbf{A}}^{-1} \mathbf{K}
$$

and represents the resulting electro-mechanical concentration matrix of the GMC model that expresses the local (subcells) variables, $\mathbf{X}_{\mathbf{b}}$, in terms of the global (average) variables, $\overline{\mathbf{X}}$. Consequently, eqn.(41) is usually referred to as localization.

Let the concentration matrix $\mathrm{A}$ be partitioned into $N_{\alpha} N_{\beta} N_{\gamma}$ 9-order square submatrices in the form

$$
\mathbf{A}=\left[\begin{array}{l}
\mathbf{A}^{(111)} \\
\vdots \\
\mathbf{A}^{\left(N_{\alpha} N_{\beta} N_{\gamma}\right)}
\end{array}\right]
$$

It follows from eqn.(41) that

$$
\mathbf{X}^{(\alpha \beta \gamma)}=\mathbf{A}^{(\alpha \beta \gamma)} \overline{\mathbf{X}}
$$

Equation (43) expresses the average strain in the subcell $(\alpha \beta \gamma)$ in terms of the uniform overall strain and electric field (i.e., the applied macrostrain and macroelectric field). 
Substitution of eqn.(43) into (12) yields

$$
\mathbf{Y}^{(\alpha \beta \gamma)}=\mathbf{Z}^{(\alpha \beta \gamma)} \mathbf{A}^{(\alpha \beta \gamma)} \overline{\mathbf{X}}
$$

Consequently, in conjunction with the averaging procedure given by (14), the following effective constitutive relations of the piezoelectric composite can be established

$$
\overline{\mathbf{Y}}=\mathbf{Z}^{*} \overline{\mathbf{X}}
$$

where the effective elastic stiffness, piezoelectric and dielectric matrix $\mathbf{Z}^{*}$ of the composite is given by

$$
\mathbf{Z}^{*}=\frac{1}{d h l} \sum_{\alpha=1}^{N_{\alpha}} \sum_{\beta=1}^{N_{\beta}} \sum_{\gamma=1}^{N_{\gamma}} d_{\alpha} h_{\beta} l_{\gamma} \mathbf{Z}^{(\alpha \beta \gamma)} \mathbf{A}^{(\alpha \beta \gamma)}
$$

The structure of the square $9-$ th order effective matrix $\mathbf{Z}^{*}$ is of the form

$$
\mathbf{Z}^{*}=\left[\begin{array}{ll}
\mathbf{C}^{*} & \mathrm{e}^{* \mathrm{t}} \\
\mathrm{e}^{*} & -\lambda^{*}
\end{array}\right]
$$

Thus this micromechanically derived relation readily provides the effective 6 by 6 stiffness matrix $\mathbf{C}^{*}$, the effective piezoelectric 3 by 6 matrix $\mathrm{e}^{*}$, and the effective dielectric 3 by 3 matrix $\lambda^{*}$.

\subsection{Effective thermal and pyroelectric coefficients}

In establishing the overall behavior of the piezoelectric composite, isothermal conditions were assumed (see eqn. (12)). Although it is possible to use the same GMC model to predict the effective thermal and pyroelectric constants, it is more convenient to utilize Levin's (1967) result to establish these constants. This approach was previously used by Dunn (1993) to establish these constants in conjunction with other micromechanical models. It was also utilized by Aboudi (1995) to verify that the effective thermal coefficients and the overall inelastic strains as predicted by GMC are in conformity with the corresponding prediction by Levin's method.

To this end referring to eqns. (6) and (7), let us define the following vector of thermal stresses and pyroelectric constants in the material filling the subcell $(\alpha \beta \gamma)$ :

$$
\Gamma^{(\alpha \beta \gamma)}=\left(\Lambda_{1}^{(\alpha \beta \gamma)}, \Lambda_{2}^{(\alpha \beta \gamma)}, \Lambda_{3}^{(\alpha \beta \gamma)}, \Lambda_{4}^{(\alpha \beta \gamma)}, \Lambda_{5}^{(\alpha \beta \gamma)}, \Lambda_{6}^{(\alpha \beta \gamma)}, p_{1}^{(\alpha \beta \gamma)}, p_{2}^{(\alpha \beta \gamma)}, p_{3}^{(\alpha \beta \gamma)}\right)
$$


The corresponding global (effective) vector is defined by

$$
\Gamma^{*}=\left(\Lambda_{1}^{*}, \Lambda_{2}^{*}, \Lambda_{3}^{*} \Lambda_{4}^{*}, \Lambda_{5}^{*}, \Lambda_{6}^{*}, p_{1}^{*}, p_{2}^{*}, p_{3}^{*}\right)
$$

According to Levin's result, the relation between $\Gamma^{(\alpha \beta \gamma)}$ and $\Gamma^{*}$ is given in terms of the electro-mechanical concentration matrices $\mathbf{A}^{(\alpha \beta \gamma)}$ as follows.

$$
\Gamma^{*}=\frac{1}{d h l} \sum_{\alpha=1}^{N_{\alpha}} \sum_{\beta=1}^{N_{\beta}} \sum_{\gamma=1}^{N_{\gamma}} d_{\alpha} h_{\beta} l_{\gamma}\left[\mathbf{A}^{(\alpha \beta \gamma)}\right]^{T} \Gamma^{(\alpha \beta \gamma)}
$$

where $\left[\mathbf{A}^{(\alpha \beta \gamma)}\right]^{T}$ is the transpose of $\mathbf{A}^{(\alpha \beta \gamma)}$. The above relation provides the effective thermal stress $\Lambda^{*}$ and pyroelectric $\mathbf{p}^{*}$ vectors of the composite.

Finally, the effective coefficients of thermal expansion $\alpha_{i}^{*}$ and the associated pyroelectric constants $P_{i}^{*}$ of the composite can be assembled into the vector:

$$
\Omega^{*}=\left(\alpha_{1}^{*}, \alpha_{2}^{*}, \alpha_{3}^{*}, \alpha_{4}^{*}, \alpha_{5}^{*}, \alpha_{6}^{*}, P_{1}^{*}, P_{2}^{*}, P_{3}^{*}\right)
$$

This vector is given by:

$$
\boldsymbol{\Omega}^{*}=\mathbf{Z}^{*-1} \Gamma^{*}
$$

It should be mentioned that a similar relation holds for the local quantities, namely for the the coefficients of thermal expansion and the associated pyroelectric constants of the materials filling the subcells. In such a case $\mathbf{Z}^{*}$ and $\Gamma^{*}$ should be replaced by $\mathbf{Z}^{(\alpha \beta \gamma)}$ and $\Gamma^{(\alpha \beta \gamma)}$, respectively.

\section{Piezothermoelastic Laminated Plate}

The micromechanically established effective constitutive relations

$$
\bar{\sigma}_{i j}=C_{i j k l}^{*} \bar{\epsilon}_{k l}-e_{k i j}^{*} \bar{E}_{k}-\Lambda_{i j}^{*} \Delta T
$$

can be utilized to derive the basic equations for piezothermoelastic laminated plates in conjunction with the classical plate theory. To this end, let us consider, $N$ layers of orthorhombic piezoelectric materials. For this type of material, the constitutive equations, eqns. (53), that describes the behavior of a single lamina, can be written in the following form (Tauchert, 
1992).

$$
\begin{aligned}
& \left\{\begin{array}{c}
\bar{\sigma}_{11} \\
\bar{\sigma}_{22} \\
\bar{\sigma}_{33} \\
\bar{\sigma}_{23} \\
\bar{\sigma}_{13} \\
\bar{\sigma}_{12}
\end{array}\right\}=\left[\begin{array}{cccccc}
c_{11}^{*} & c_{12}^{*} & c_{13}^{*} & 0 & 0 & 0 \\
& c_{22}^{*} & c_{23}^{*} & 0 & 0 & 0 \\
& & c_{33}^{*} & 0 & 0 & 0 \\
& & & c_{44}^{*} & 0 & 0 \\
& & & & c_{55}^{*} & 0 \\
& & & & & \\
\text { sym. } & & & & & c_{66}^{*}
\end{array}\right]\left\{\begin{array}{c}
\bar{\epsilon}_{11} \\
\bar{\epsilon}_{22} \\
\bar{\epsilon}_{33} \\
2 \bar{\epsilon}_{23} \\
2 \bar{\epsilon}_{13} \\
2 \bar{\epsilon}_{12}
\end{array}\right\} \\
& -\left[\begin{array}{ccc}
0 & 0 & e_{31}^{*} \\
0 & 0 & e_{32}^{*} \\
0 & 0 & e_{33}^{*} \\
0 & e_{24}^{*} & 0 \\
e_{15}^{*} & 0 & 0 \\
0 & 0 & 0
\end{array}\right]\left\{\begin{array}{c}
\bar{E}_{1} \\
E_{2} \\
\bar{E}_{3}
\end{array}\right\}-\left\{\begin{array}{c}
\Lambda_{1}^{*} \\
\Lambda_{2}^{*} \\
\Lambda_{3}^{*} \\
0 \\
0 \\
0
\end{array}\right\} \Delta T
\end{aligned}
$$

When the behavior of the lamina is referred to the plate system of coordinates $x, y, z=x_{3}$, and plane stress conditions are imposed, the following constitutive relations can be obtained from (54).

$$
\left\{\begin{array}{c}
\sigma_{x x} \\
\sigma_{y y} \\
\sigma_{x y}
\end{array}\right\}=[\bar{Q}]\left\{\begin{array}{c}
\epsilon_{x x} \\
\epsilon_{y y} \\
2 \epsilon_{x y}
\end{array}\right\}-\left[\begin{array}{lll}
0 & 0 & \bar{e}_{31} \\
0 & 0 & \bar{e}_{32} \\
0 & 0 & \bar{e}_{36}
\end{array}\right]\left\{\begin{array}{c}
E_{x} \\
E_{y} \\
E_{z}
\end{array}\right]-\left\{\begin{array}{c}
\Lambda_{x} \\
\Lambda_{y} \\
\Lambda_{x y}
\end{array}\right\} \Delta T
$$

In this equation $\left[\bar{Q}_{i j}\right]$ are the reduced stiffness coefficients which are given in terms of the transformed effective stiffnesses $\left[\bar{c}_{i j}^{*}\right]$ by the standard relations (see Herakovich (1998)):

$$
\bar{Q}_{i j}=\bar{c}_{i j}^{*}-\frac{\bar{c}_{i 3}^{*} \bar{c}_{3 j}^{*}}{\bar{c}_{33}^{*}} \quad i, j=1,2,6
$$

The transformed piezoelectric constants and thermal stress coefficients are given by

$$
\begin{gathered}
\bar{e}_{31}^{*}=e_{31}^{*} c^{2}+e_{32}^{*} s^{2}, \bar{e}_{32}^{*}=e_{31}^{*} s^{2}+e_{32}^{*} c^{2}, \bar{e}_{36}^{*}=\left(e_{31}^{*}-e_{32}^{*}\right) s c \\
\Lambda_{x}=\Lambda_{1}^{*} c^{2}+\Lambda_{2}^{*} s^{2}, \Lambda_{y}=\Lambda_{1}^{*} s^{2}+\Lambda_{2}^{*} c^{2}, \Lambda_{x y}=\left(\Lambda_{1}^{*}-\Lambda_{2}^{*}\right) s c
\end{gathered}
$$

with $c$ and $s$ denoting the sin and cos of the angle between the $x$ and $x_{1}$ axis. 
The stress resultants, $\mathbf{N}$, and moments, $\mathbf{M}$, of the laminate can be readily obtained in the form:

$$
\left\{\begin{array}{l}
\mathrm{N} \\
\mathrm{M}
\end{array}\right\}=\left[\begin{array}{ll}
\mathrm{A} & \mathrm{B} \\
\mathrm{B} & \mathrm{D}
\end{array}\right]\left\{\begin{array}{l}
\epsilon^{0} \\
\kappa
\end{array}\right\}-\left\{\begin{array}{l}
\mathrm{N}^{E} \\
\mathrm{M}^{E}
\end{array}\right\}-\left\{\begin{array}{l}
\mathrm{N}^{T} \\
\mathrm{M}^{T}
\end{array}\right\}
$$

where $\mathrm{A}, \mathrm{B}$ and $\mathrm{D}$ denote the standard extensional, coupling and bending stiffnesses, and, $\epsilon^{0}$ and $\kappa$ represent the middle surface strains and curvatures, respectively. In eqn.(56), the electric resultants, $\mathbf{N}^{E}$, and moments, $\mathbf{M}^{E}$, are defined by

$$
\begin{gathered}
\mathbf{N}^{E}=\left\{\begin{array}{c}
N_{x}^{E} \\
N_{y}^{E} \\
N_{x y}^{E}
\end{array}\right\}=\sum_{k=1}^{N}\left\{\begin{array}{c}
\bar{e}_{31} \\
\bar{e}_{32} \\
\bar{e}_{36}
\end{array}\right\}_{k}\left(E_{z}\right)_{k}\left(h_{k}-h_{k-1}\right) \\
\mathbf{M}^{E}=\left\{\begin{array}{c}
M_{x}^{E} \\
M_{y}^{E} \\
M_{x y}^{E}
\end{array}\right\}=\frac{1}{2} \sum_{k=1}^{N}\left\{\begin{array}{c}
\bar{e}_{31} \\
\bar{e}_{32} \\
\bar{e}_{36}
\end{array}\right\}_{k}\left(E_{z}\right)_{k}\left(h_{k}^{2}-h_{k-1}^{2}\right)
\end{gathered}
$$

where $h_{k}$ denotes the thickness of the $k$ th lamina. The thermal resultants, $\mathbf{N}^{T}$, and moments, $\mathbf{M}^{T}$, are given by

$$
\begin{gathered}
\mathbf{N}^{T}=\left\{\begin{array}{c}
N_{x}^{T} \\
N_{y}^{T} \\
N_{x y}^{T}
\end{array}\right\}=\sum_{k=1}^{N}\left\{\begin{array}{c}
\Lambda_{x} \\
\Lambda_{y} \\
\Lambda_{x y}
\end{array}\right\}_{k} \Delta T\left(h_{k}-h_{k-1}\right) \\
\mathbf{M}^{T}=\left\{\begin{array}{c}
M_{x}^{T} \\
M_{y}^{T} \\
M_{x y}^{T}
\end{array}\right\}=\frac{1}{2} \sum_{k=1}^{N}\left\{\begin{array}{c}
\Lambda_{x} \\
\Lambda_{y} \\
\Lambda_{x y}
\end{array}\right\}_{k} \Delta T\left(h_{k}^{2}-h_{k-1}^{2}\right)
\end{gathered}
$$

\section{Results}

In order to assess the reliability of the effective constants of piezoelectric composites predicted by GMC, comparisons with other micromechanical methods and with measured data must be performed. Dunn and Taya (1993) and Dunn (1993) conducted extensive study of the effective constants of piezoelectric composites based on various micromechanical models, and performed several comparisons with experimental results. They concluded that the Mori 
and Tanka (1973) scheme (MT) is the recommended one for application. Consequently, we present here comparisons with MT predictions as given by Dunn and Taya (1993) and Dunn (1993) in order to verify the validity of GMC predictions. To this end piezoelectric materials with hexagonal symmetry in which the axis of symmetry is aligned in the 3-direction are considered. The constitutive law of the material that possesses this type of symmetry is given by eqn. (54) when

$$
c_{11}^{*}=c_{22}^{*}, c_{23}^{*}=c_{13}^{*}, \quad c_{44}^{*}=c_{55}^{*}, c_{66}^{*}=\frac{1}{2}\left(c_{11}^{*}-c_{12}^{*}\right), e_{31}^{*}=e_{32}^{*}, e_{24}^{*}=e_{15}^{*} .
$$

Tables 1-3 present the properties of several piezoelectric materials together with the properties of isotropic polymers.

In Fig. 2 comparisons between GMC and MT predictions are given for the effective piezoelectric constants of a porous $P Z T-7 A$ ceramic. Clearly, good agreement is observed.

Let us define the effective compliance matrix $\mathbf{S}^{*}$ to be the inverse of the effective stiffness matrix $\mathrm{C}^{*}$. Figure 3 shows the effective elastic compliances $S_{11}^{*}+S_{12}^{*}$ of a piezoelectric $P Z T-7 A$ ceramic long fiber reinforcing an epoxy matrix. Here the GMC and MT predictions coincide.

Another set of effective piezoelectric coefficients $d_{i j}^{*}$ (which appear when the constitutive relation of the piezoelectric material is formulated as strains expressed in terms of stresses and electric field) are defined as follows

$$
d_{i j}^{*}=e_{i k}^{*} S_{k j}^{*}
$$

In Fig. 4 the above effective piezoelectric constants as predicted by GMC and MT are shown for a $P Z T-5$ ceramic matrix reinforced by long polymer fibers. The agreement is excellent for $d_{33}$ and satisfactory for $d_{h}$.

Figures 5-7 present the effective coefficients of thermal expansion and the effective pyroelectric constants of a polymeric matrix reinforced by long piezoelectric fibers. The predictions of GMC and MT coincide, as expected since both micromechanical approaches use Levin's method.

Figures 8 and 9 present the effective coefficients of thermal expansion and the effective pyroelectric constant of a polymeric matrix reinforced by piezoelectric particles. The predictions of GMC and MT coincide for the effective thermal properties shown in Fig. 8 and 
closely correspond for the effective pyroelectric coefficient given in Fig. 9. For the same composite system, the effective pyroelectric constant $-P_{3}^{*} / Z_{99}^{*-1}$ as predicted by GMC and MT is shown in Fig. 10. Here, slight differences can be observed in the vicinity of very high volume fraction where steep gradients occur.

Previous analyses carried out by various researchers (see the update review by Aboudi (1996)) have shown that GMC is a reliable and efficient micromechanical model for predicting the behavior of various types of composite materials. Based on the present results, it can be concluded that GMC is also a reliable micromechanical tool for the prediction of the effective parameters of piezoelectric multiphase composites.

\section{Conclusions}

Micromechanical prediction of the effective elastic, piezoelectric, thermal and pyroelectric constants of piezoelectric composites by the generalized method of cells (GMC) have been derived. These constants were verified by comparisons with those predicted using the Mori-Tanaka (MT) model and found to be in good agreement. An advantage of the present model, over other micromechanical formulations, is its ability to predict the behavior of multiphased piezoelectric materials and composites. In this connection it should be emphasized that the Mori-Tanaka method has been previously shown by Ferrari (1991) to yield an asymmetric effective stiffness tensor in general, which obviously is a shortcoming of the method. Additionally, GMC can be used, to investigate other effects (as previously shown by several investigators), such as; the effect of inelasticity of the host material, the effect of weak bonding between the constituents, and the effect of fiber distribution and architecture on the overall response of piezoelectric composites. Also, the established macromechanical relations can be readily linked to a structural analysis for the analysis of piezoelectric composite structures. 


\section{Acknowledgment}

The author gratefully acknowledges the support of the Diane and Arthur Belfer chair of Mechanics and Biomechanics. Also special thanks go to Dr. S. M. Arnold, NASA-Lewis Research Center, for his suggestion to initiate this investigation, for several fruitful discussions during the course of this work and for his assistance with the preparation of this report.

Table 1. Elastic material properties.

\begin{tabular}{cccccc}
\hline Material & $C_{11}(G P a)$ & $C_{12}(G P a)$ & $C_{13}(G P a)$ & $C_{33}(G P a)$ & $C_{44}(G P a)$ \\
\hline$P Z T-7 A$ & 148 & 76.2 & 74.2 & 131 & 25.4 \\
BaTiO $_{3}$ & 150 & 66 & 66 & 146 & 44 \\
Epoxy & 8 & 4.4 & 4.4 & 8 & 1.8 \\
Polymer & 3.86 & 2.57 & 2.57 & 3.86 & 0.64 \\
\hline
\end{tabular}

Table 2. Electric material properties.

\begin{tabular}{cccccc}
\hline Material & $e_{15}\left(\mathrm{C} / \mathrm{m}^{2}\right)$ & $e_{31}\left(\mathrm{C} / \mathrm{m}^{2}\right)$ & $e_{33}\left(\mathrm{C} / \mathrm{m}^{2}\right)$ & $\lambda_{1}\left(10^{-9} \mathrm{C} / \mathrm{Vm}\right)$ & $\lambda_{3}\left(10^{-9} \mathrm{C} / \mathrm{Vm}\right)$ \\
\hline PZT-7A & 9.2 & -2.1 & 9.5 & 4.07 & 2.07 \\
BaTiO $_{3}$ & 11.4 & -4.35 & 17.5 & 9.86 & 11.15 \\
Epoxy & 0 & 0 & 0 & 0.037 & 0.037 \\
Polymer & 0 & 0 & 0 & 0.079 & 0.079 \\
\hline
\end{tabular}

Table 3. Coefficients of thermal expansion and pyroelectric material properties.

\begin{tabular}{cccc}
\hline Material & $\alpha_{1}\left(10^{-6} / K\right)$ & $\alpha_{3}\left(10^{-6} / K\right)$ & $P_{3}\left(10^{5} N / C K\right)$ \\
\hline BaTiO $_{3}$ & 8.53 & 1.99 & 0.133 \\
Epoxy & 60 & 60 & 0 \\
\hline
\end{tabular}




\section{References}

Aboudi, J. (1989) Micromechanical Analysis of Composites by the Method of Cells. Appl. Mech. Rev. 42, 193-221.

Aboudi, J. (1991) Mechanics of Composite Materials: A Unified Micromechanical Approach. Elsevier, Amsterdam.

Aboudi, J. (1995) Micromechanical Analysis of Thermoinelastic Multiphase Short-fiber Composites. Composites Eng. 5, 839-850.

Aboudi, J. (1996) Micromechanical Analysis of Composites by the Method of Cells Update. Appl. Mech. Rev. 49, S83-S91.

Carman, G.P., Cheung, K.S. and Wang, D. (1995) Micro-mechanical Model of a Composite Containing a Conservative Nonlinear Electro-magneto-thermo-mechanical Material. J. Intell. Material Systems \& Structures 6, 691-699.

Dunn, M.L. (1993) Micromechanics of Coupled Electroelastic Composites: Effective Thermal Expansion and Pyroelectric Coefficients. J. Appl. Physics 73, 5131-5140.

Dunn, M.L. and Taya M. (1993) Micromechanics Prediction of the Effective Electroelastic Moduli of Piezoelectric Composites. Int. J. Solids \& Structures 30, 161-175.

Ferrari, M. (1991) Asymmetry and the High Concentration Limit of the Mori-Tanaka Effective Medium Theory. Mech. Materials 11, 251-256.

Gandhi M.V. and Thompson B.S. (1992) Smart Materials and Structures. Chapman \& Hall.

Herakovich, C.T. (1998) Mechanics of Fibrous Composites. Wiley, New York.

Jain, A.K. and Sirkis, J.S. (1994) Continuum Damage Mechanics in Piezoelectric Ceramics. AD-vol. 45/MD-vol. 54, 47-58, ASME. 
Jonnalagadda, K.D., Blandford, G.E. and Tauchert, T.R. (1994) Piezothermoelastic Composite Plate Analysis Using First-order Shear Deformation Theory. Computers \& Structures 51 79-89.

Mori, T., and Tanaka, K. (1973), Average Stresses in Matrix and Average Energy of Materials with Misfitting Inclusions, Acta Metall. 21, 571-574.

Moulson, A.J. and Herbert, J.M. (1990), Electroceramics. Chapman \& Hall.

Levin, V.M. (1967) On the Coefficients of Thermal Expansion of Heterogeneous Materials. Mech. Solids 2, 58-61.

Parton, V.Z. and Krudryavtsev, B.Z. (1988) Electromagnetoelasticity. Gordon and Breach Science Publisher.

Tauchert, T.R. (1992), Piezothermoelastic Behavior of Laminated Plate. J. Thermal Stresses 15, 25-37. 


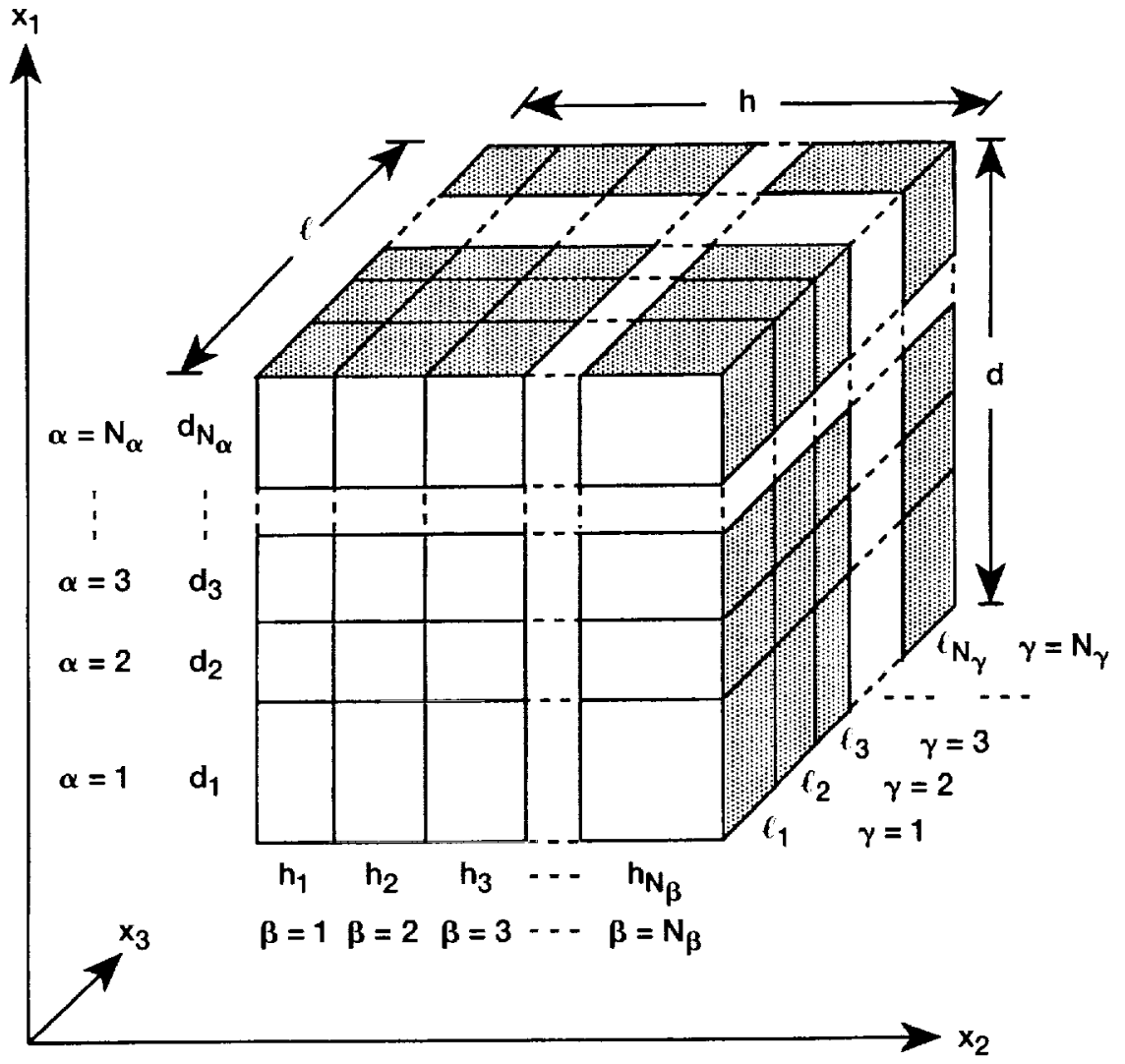

Figure 1.-A repeating cell in GMC consisting of $N_{\alpha}, N_{\beta}$ and $N_{\gamma}$ subcells in the 1,2 and 3 directions, respectively.

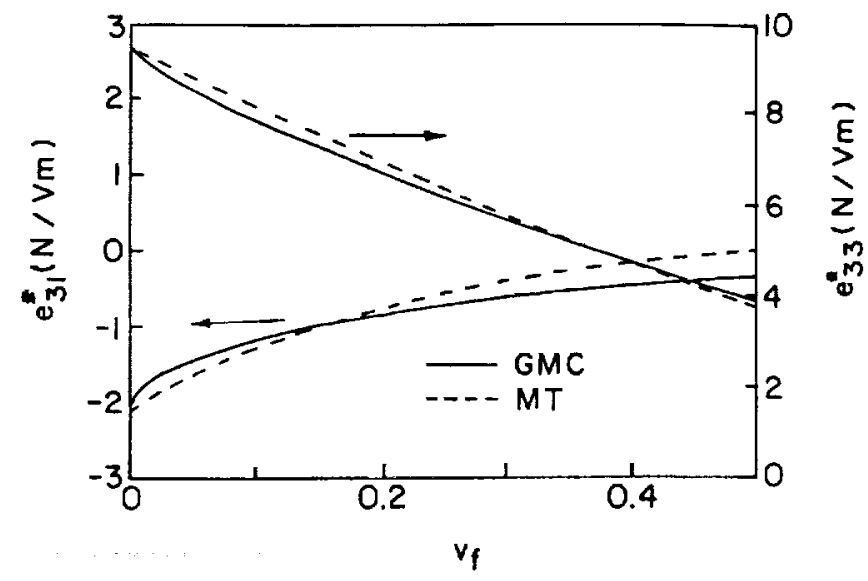

Figure 2.-Comparison between the effective piezoelectric moduli $e_{3 i}^{*}$ as predicted by GMC and MT for a porous PZT - 7A ceramic as a function of the porosity volume fraction. 


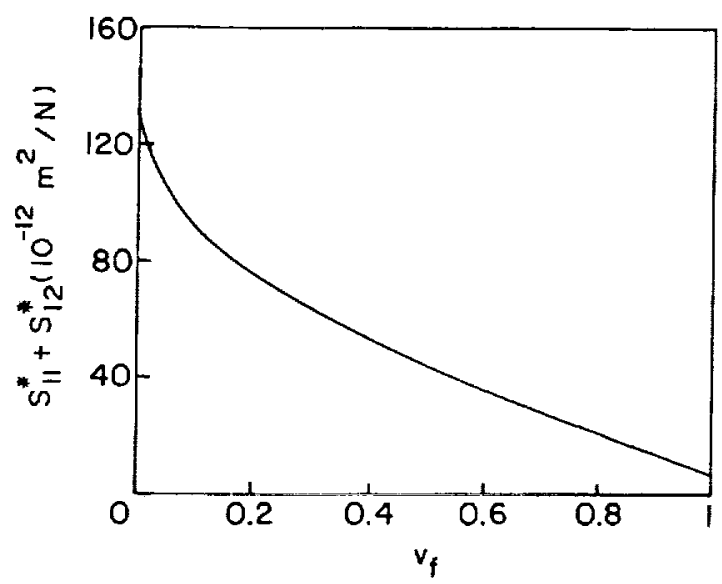

Figure 3.-The effective elastic compliances $\mathrm{S}_{11}^{*}+$ $S_{12}^{*}$ of an epoxy matrix reinforced by continuous PZT - 7A fiber as a function of the fiber volume ratio. The predictions of GMC and MT coincide.

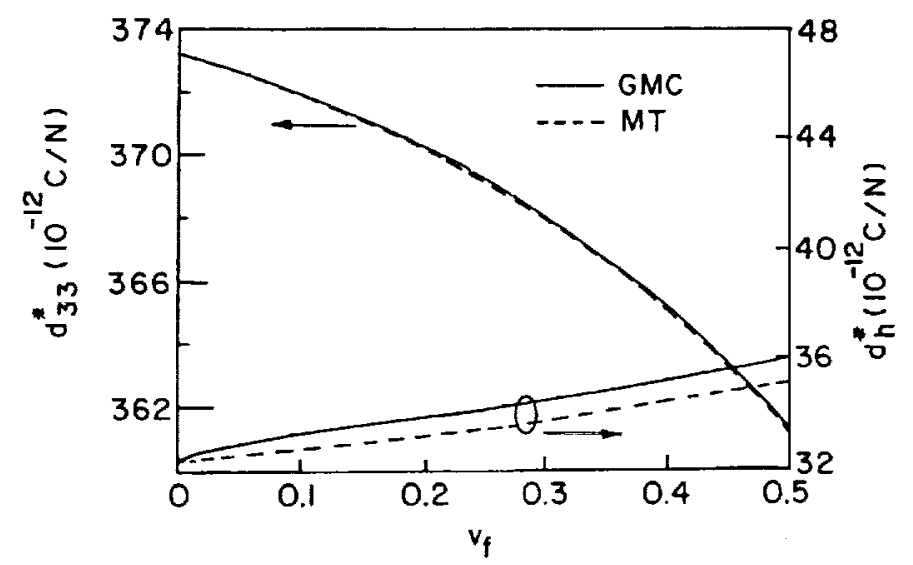

Figure 4.-Comparison between the effective piezoelectric moduli $d_{33}^{*}$ and $d_{h}^{*}=d_{31}^{*}+d_{32}^{*}+d_{33}$ as predicted by GMC and MT for a PZT - 5 ceramic matrix reinforced by a continuous polymer as a function of the fiber volume fraction.

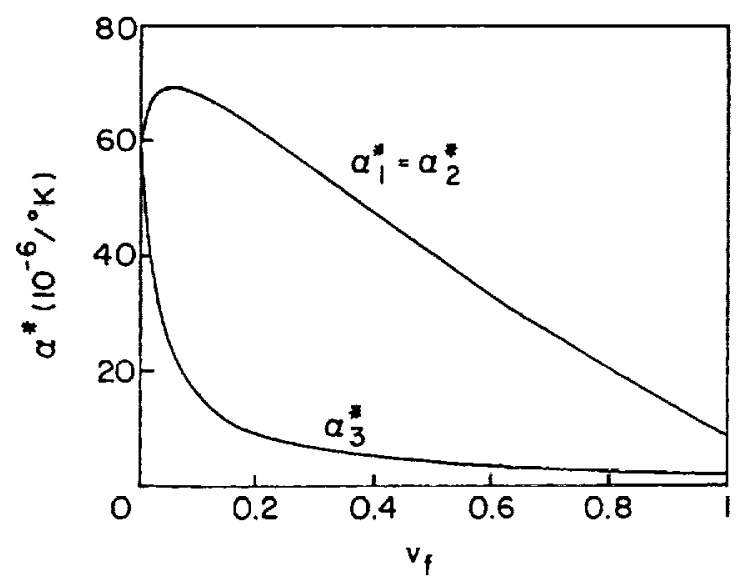

Figure 5.-The effective coefficients of thermal expansion $\alpha_{1}^{*}=\alpha_{2}^{*}, \alpha_{3}^{*}$ for a continuous $\mathrm{BaTiO}_{3}$ fiber reinforcing a polymer epoxy matrix as a function of the fiber volume fraction. The predictions of GMC and MT coincide.

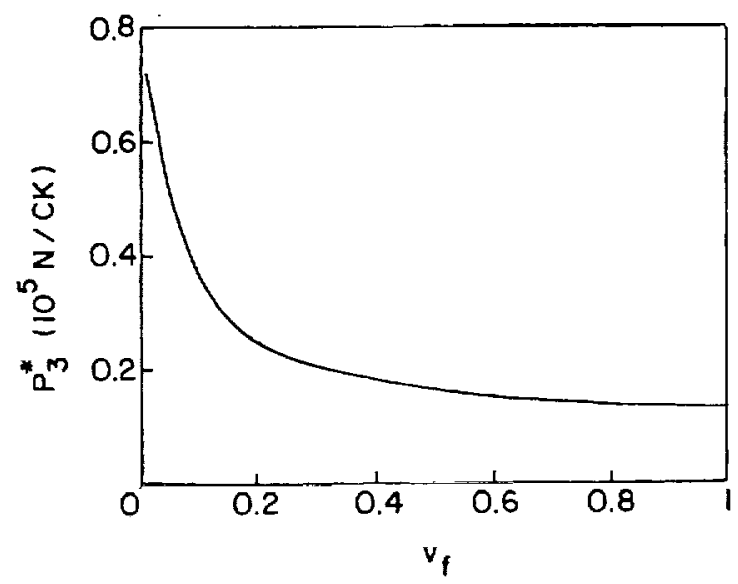

Figure 6.-The effective pyroelectric coefficients $\mathrm{P}_{3}^{\star}$, of a continuous $\mathrm{BaTiO}_{3}$ fiber reinforcing a polymer epoxy matrix as a function of the fiber volume fraction. The predictions of GMC and MT coincide. 


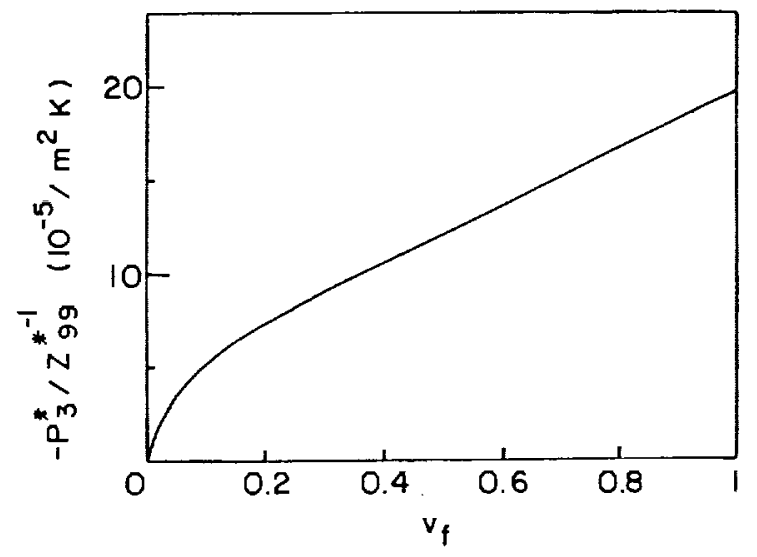

Figure 7.-The effective pyroelectric coefficients $-\mathrm{P}_{3}^{\star} / \mathrm{Z}_{99}^{\star}{ }^{-1}$ of a continuous $\mathrm{BaTiO}_{3}$ fiber reinforcing a polymer epoxy matrix as a function of the fiber volume fraction. The predictions of GMC and MT coincide.

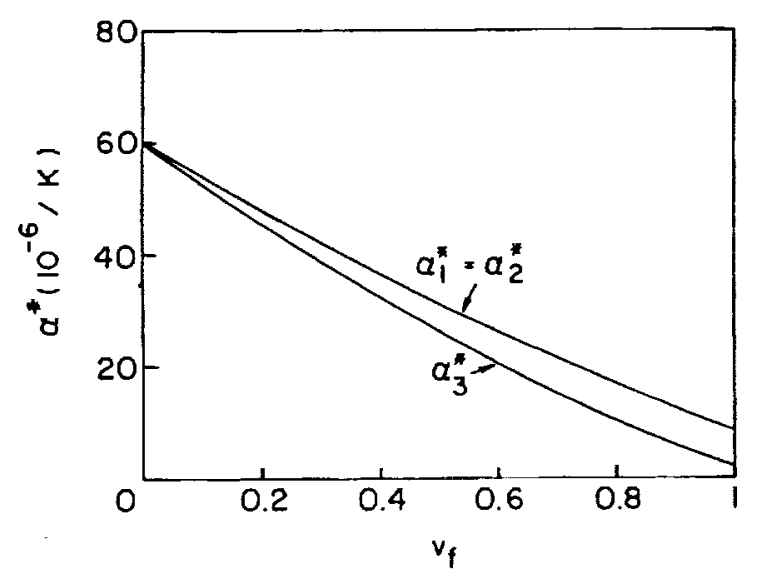

Figure 8.-The effective coefficients of thermal expansion $\alpha_{1}^{*}=\alpha_{2}^{*}, \alpha_{3}^{*}$ for $\mathrm{BaTiO}_{3}$ particles reinforcing a polymer epoxy matrix as a function of the particles volume fraction. The predictions of GMC and MT coincide.

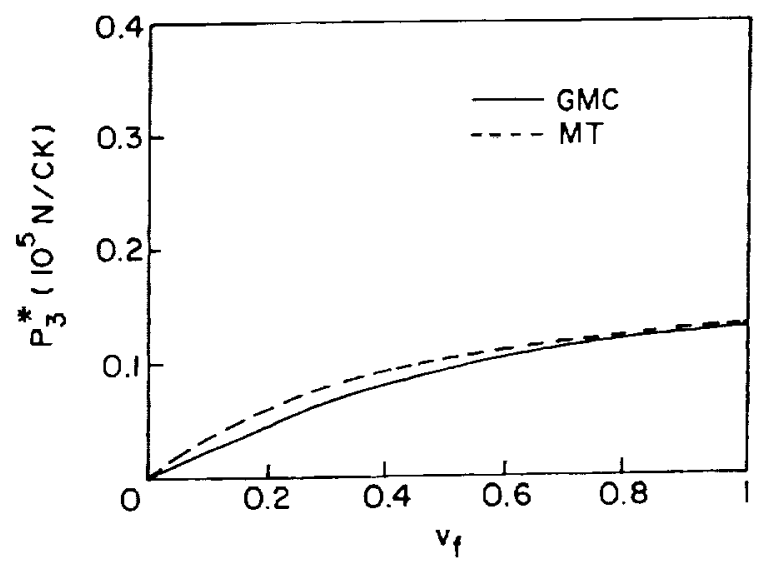

Figure 9.-Comparison between the effective pyroelectric coefficient $P_{3}^{*}$, as predicted by $G M C$ and $\mathrm{MT}$ for $\mathrm{BaTiO}_{3}$ particles reinforcing a polymer epoxy matrix as a function of the particles volume fraction.

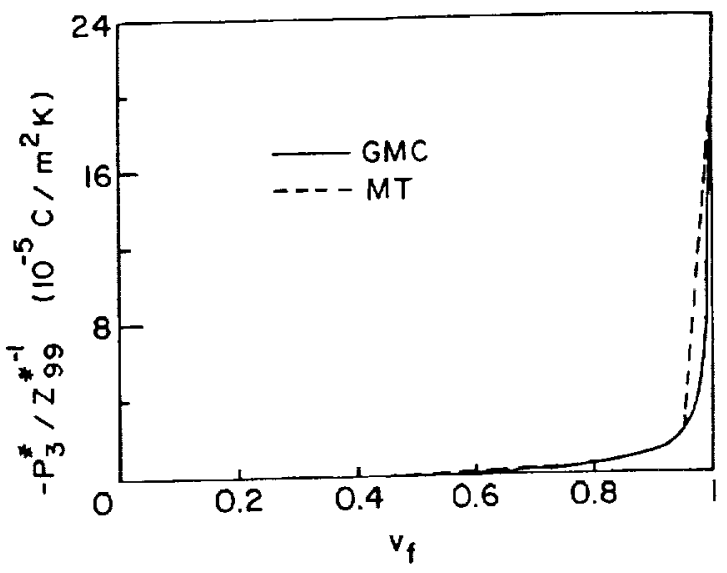

Figure 10.-Comparison between the effective pyroelectric coefficient $-\mathrm{P}_{3}^{\star} / \mathrm{Z}_{99}^{*}{ }^{-1}$, as predicted by $\mathrm{GMC}$ and $\mathrm{MT}$ for $\mathrm{BaTiO}_{3}$ particles reinforcing a polymer epoxy matrix as a function of the particles volume fraction. 


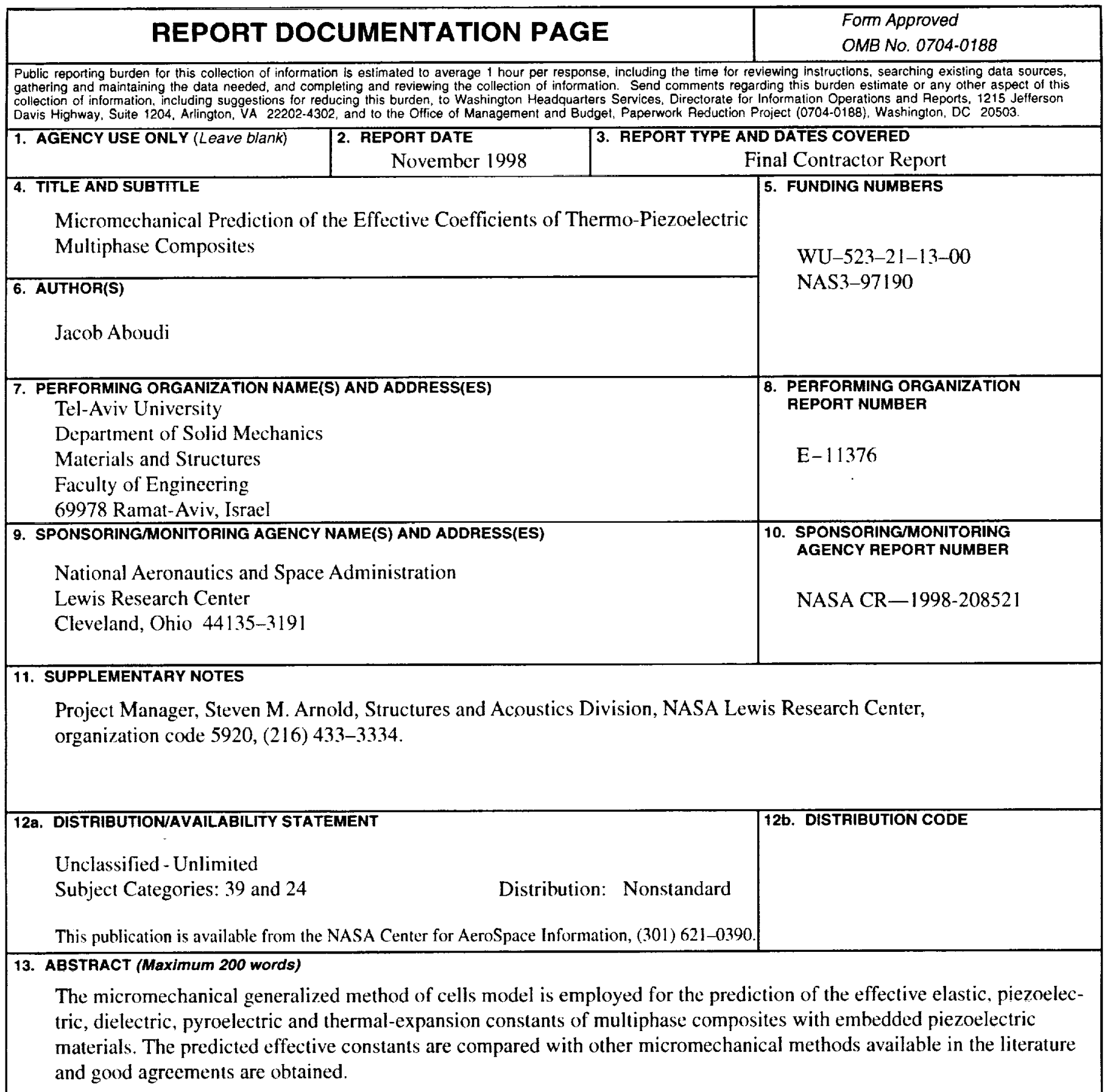

